\title{
Research on the Status Quo and Problems of the Interconnection between China's Internet Backbone Networks
}

\author{
Meijuan $\mathrm{Li}^{\mathrm{a}}$, Qianbing Xiao ${ }^{\mathrm{b}}$

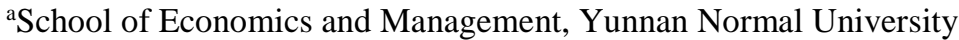 \\ Kunming and 650500, China \\ ${ }^{\mathrm{b} S c h o o l}$ of Economics and Management, Yunnan Normal University \\ Kunming and 650500, China
}

\begin{abstract}
The development of the Internet in China is a problem left behind by human beings rather than a result of market competition. In view of the huge scale difference between the dominant and vulnerable backbone networks, and for the security consideration of network, the Chinese government prohibits the transit between different networks. Therefore, the restriction of interconnection mode make the small and medium networks have to peer with the dominant network. Because of the lack of interconnection enthusiasm, the quality of Internet interconnection of the dominant backbone network is not effectively guaranteed and the Internet settlement fee is high, which seriously affect the user experience and not good for the development of the Internet industry. In addition, the lack of Internet laws and regulations and insufficient interconnection between Internet networks have seriously damaged the consumer surplus. Therefore, this paper proposes that China should introduce transit on the premise of network security, cut down settlement fees, establish monitoring system of the interconnection quality, strengthen legislation work and adhere to government regulations, which can promote the healthy development of Internet backbone network in China.
\end{abstract}

Keywords-Internet backbone network; Interconnection; Peering; Transit

\section{THE DEVELOPMENT HISTORY OF INTERCONNECTION IN CHINA'S INTERNET BACKBONE NETWORK}

On April 20 of 1994, the 64K international line of the NCFC project which connected to the Internet through Sprint Corporation of the United States was opened. It realized connection with the Internet and marked the birth of the Internet in China. In January 1995, the General Administration of Telecommunications of the Ministry of Posts and Telecommunications started to provide Internet access services for the public. In January 1996, the China Public Computer Internet (CHINANET) backbone network was established and officially opened. In October 1997, the China Public Computer Internet (CHINANET) achieved interconnection with other three Internet networks in China, namely China Science and Technology Network (CSTNET), China Education and Research Computer Network (CERNET) and China Golden Bridge Information Network (CHINAGBN), which preliminarily formed the network interconnection structure in China. Subsequently, the relevant departments of the State Council had set up 6 Internet backbone network operators, namely China Unicom, China Railcom, China Mobile, China Satellite Group Internet, China Great Wall Internet and China International Economic and trade Internet. At the end of 2001, the top 10 Internet backbone networks signed interconnection agreements and achieved cross-regional network access. The telecommunications industry has undergone a series of reforms and reorganizations. China Golden Bridge Information Network and China Satellite Group Internet were withdrawn from the major backbone operators in 2002 and 2009 respectively, and the number of 10 large backbone networks was reduced to 8 [1].

On November 27 of 2015, China Mobile Tietong, a wholly-owned subsidiary of China Mobile, acquired the target assets, business and related debts of China Railcom Group Co., Ltd. China Railcom has become a history. Since then, the main internet backbone network operators has been reduced from 8 to 7.They are China Telecom, China Unicom, China Mobile, China Education and Research Computer Network, China Science and Technology network, China International Economic and Trade Internet and China Great Wall Internet. Among them, the first 3 networks belong to profitable operators, while the latter 4 are non-profitable operators. Internet backbone network operators in China have great disparity in strength due to the differences in establishment time and investment conditions. China Telecom and China Unicom are the two largest backbone networks in China. They are also known as Tier-1 Internet backbone network, and the remaining five are Tier-2 [2]. Tables 1 and table 2 show the international export bandwidth and broadband users of major Internet backbone networks in China during 2009-2016 
TABLE I. THE INTERNATIONAL EXPORT BANDWIDTH OF MAJOR INTERNET BACKBONE NETWORK OPERATORS IN CHINA(UNIT: MBPS)

\begin{tabular}{|c|c|c|c|c|c|c|c|}
\hline years & $\begin{array}{c}\text { China } \\
\text { Telecom }\end{array}$ & $\begin{array}{c}\text { China } \\
\text { Unicom }\end{array}$ & $\begin{array}{c}\text { China } \\
\text { Mobile }\end{array}$ & $\begin{array}{c}\text { China } \\
\text { Education } \\
\text { and } \\
\text { Research } \\
\text { Computer } \\
\text { Network }\end{array}$ & $\begin{array}{c}\text { China } \\
\text { Science and } \\
\text { technology } \\
\text { network }\end{array}$ & $\begin{array}{c}\text { China } \\
\text { International } \\
\text { Economic and } \\
\text { Trade Internet }\end{array}$ & total \\
\hline 2009 & 516650.2 & 298834 & 30559 & 10000 & 10322 & 2 & 866367.2 \\
\hline 2010 & 660612.82 & 357433 & 49124 & 11655 & 18120 & 2 & 1098956.82 \\
\hline 2011 & 809881 & 466932 & 82559 & 11655 & 18500 & 2 & 1389529 \\
\hline 2012 & 1048848 & 586279 & 206563 & 35500 & 22600 & 2 & 1899792 \\
\hline 2013 & 2190878 & 850215 & 287629 & 55500 & 22600 & 2 & 3406824 \\
\hline 2014 & 2569519 & 1037023 & 390263 & 66560 & 55296 & 2 & 4118663 \\
\hline 2015 & 3223629 & 1414868 & 645073 & 61440 & 47104 & 2 & 5392116 \\
\hline 2016 & 3886527 & 1700446 & 959108 & 40960 & 53248 & 2 & 6640291 \\
\hline
\end{tabular}

It is known from table 1 that international export bandwidth is mainly provided by three profitable networks in China, and the number of bandwidth is decreasing in sequence according to the order of China Telecom, China Unicom and China Mobile. The number of international export bandwidth of nonprofitable networks is small, which is basically negligible. In terms of the number of international export bandwidth, China Telecom and China Unicom have an absolute advantage over China Mobile. It can be seen that the Internet backbone network market in China has strong asymmetry, and there is a wide disparity in the scale between dominant internet backbone network China Telecom, China Unicom and other networks.

\section{THE CURRENT SitUATION OF INTERCONNECTION \\ BETWEEN INTERNET BACKBONE NETWORKS IN CHINA}

At present, the interconnection mode of Internet backbone network in China are mainly NAP and peering. Before the establishment of the NAP, the exchange of traffic between backbone networks in China was mainly through peering. Subsequently, in order to solve the problem of insufficient in interconnection bandwidth and difficult in reaching peering protocol, the Ministry of Information Industry established the first NAP in Beijing in March 2000. At the end of 2001, the NAP exchange center in Guangzhou and Shanghai was officially established and operated. As of the opening of Fuzhou network access point in August 2017, China have built 10 new NAPs based on three original NAPs that was built in Beijing, Guangzhou and Shanghai. The new NAPs are distributed in Chengdu, Xi'an, Wuhan, Shenyang, Nanjing, Chongqing, Zhengzhou, Hangzhou, Guiyang and Fuzhou, which make the speed of network transmission greatly improved[3]. In addition, for security considerations, China stipulate that the backbone operators can not provide transit and international transit services for other backbone networks in addition to providing transit service to small ISP.
In China, China Telecom and China Unicom that are the dominant backbone networks access to the Internet through bill-and-keep peering, the vulnerable backbone networks at the second-tier can access to the Internet through NAP and paid peering. Because the NAP is far from meeting the requirements of the Internet backbone network for the traffic transmission, each vulnerable backbone network still need to access to the

Internet through paid peering. At this time, there is no competition between the two dominant backbone networks (China Telecom and China Unicom) in providing network access to the vulnerable backbone networks. The dominant backbone networks are in a dominant position in commercial negotiations and have absolute control over the price of peering. On the one hand, the multi-home behaviors of the vulnerable backbone network increase the access cost [4]. On the other hand, it has increased the possibility of the collusion that dominant backbone networks reduce the quality of interconnection. The prohibition of transit and international transit makes the vulnerable backbone network in the passive position on the choice of Interconnection mode, which make the monopoly power of the dominant backbone networks in China continuously strengthened and the status of the vulnerable backbone network further weakened. 


\section{The MAIN PRoblems Of INTERCONNECTION BETWEEN INTERNET BACKBONE NETWORKS IN CHINA}

In recent years, the achievements of Internet backbone network in China can not be underestimated. However, due to the special market structure of Internet backbone network, there are a series of problems that need to be solved in the development process.

\section{A. The dominant backbone network lacks enthusiasm of interconnection, which lead to lower interconnection quality}

There are two interconnection modes between backbone networks in China, namely NAP and peering. Due to the problems of poor service quality and traffic congestion in the NAP, the backbone networks usually need to peer with the dominant backbone networks besides accessing to the NAP as required to meet the need of cosumers. A lot of traffic need to be transported in the process of interconnection among the dominant and vulnerable backbone networks. The distance of traffic transmission is long, which makes the benefit obtained by the dominant backbone network sometimes not make up for its cost. The lack of enthusiasm for the dominant backbone network often requires the government's coercive power to interconnect with the vulnerable backbone network. In addition, as the backbone network has competition in the end user market, the quality of interconnection will affect its share and profit in the user market [5]. Generally speaking, the dominant backbone network tends to reduce the quality of interconnection and lacks expansion incentives, which makes end users not experience high-quality content and services at all. In recent years, user's complaints have often occurred.

\section{B. The interconnection mode is single, the settlement fee between the backbone networks is relatively high}

Due to safety concerns, China prohibits backbone network interconnected through transit each other. Therefore, the vulnerable backbone network can only be connected to the two dominant backbone networks through paid peering to join the Internet. The single interconnection mode also makes the dominant backbone network lack competition from the domestic and foreign backbone networks for interconnection access, and can easily maintain its own market forces, leading to a strong monopoly. In the process of interconnecting with the dominant backbone network, the vulnerable backbone network usually needs to pay high settlement fee in order to motivate the dominant backbone network to provide higher quality of interconnection, and the settlement fee has always been high. According to statistics, the settlement fee between backbone networks in China is about 3 to 4 times that of the average level in developed countries. In addition, the dominant backbone network adopts discriminatory pricing while charging high settlement fees. A high settlement fee is charged for a competitive network, while a low price is preferentially given to a non-competitive network. This is extremely unfair to competitive networks. Competitive networks pass on high settlement fees to consumers, which damages the interests of consumers [6].

\section{The existing interconnection system is not conducive to the healthy development of the Internet industry}

The "13th Five-Year Plan" clearly states that China must develop a modern Internet industry system and implement the "Internet $+"$ action plan to promote the extensive use of the Internet, drive the transformation of production patterns and organizational mode, form a networked, intelligent, serviceoriented and collaborative new forms of industrial development. But in the current interconnection system, the modern Internet industry system is difficult to form. On the one hand, due to the existence of two dominant backbone operators, a large number of end users are monopolized by China Telecom and China Unicom. When users of other networks visit the contents of two dominant backbone networks, problems that slow web pages are often encountered, which affects users experience. Internet content providers have to connect with multiple backbone networks so that each Internet user can experience the same service. However, it has greatly increased costs and hindered the development of content providers [7]. On the other hand, under the existing interconnection system in China, the problems such as high settlement fees, unreasonable settlement methods and high fees for broadband access, have become a stumbling block for the development of the Internet industry in China, which added extra cost to Internet companies. The reform of the interconnection system needs to be implemented as soon as possible.

\section{The relevant legal system is incomplete}

In recent years, the Internet informationization in China has developed rapidly. China has gradually developed into a big network nation, but the legal construction which is closely related to the development of the Internet remains to be improved. Up to now, China has promulgated a series of administrative regulations and regulatory framework that represented by the "Regulation of Telecommunications", and has clearly defined the market access, universal services, charges and resources. However, China has not yet formed a law for Internet interconnection, and the settlement procedure is unreasonable, which brought the accusations of the backbone network operators. For example, China Telecom believes that it is larger than China Unicom in terms of scale and insists on unilateral settlement. While China Unicom believes that it is equivalent to China Telecom in terms of scale and both occupy certain market forces in the north and the south respectively and insists on the mode of Sender Keep All. The final result is that the quality of cross-network communications is low and consumer interests are damaged. In addition, the Internet regulation laws are still not perfect, and some operators even take vicious competition in order to take the lead in seizing users, such as broadband customer premises network. In the past, in order to monopolize the management rights of broadband of the community, the operator not only paid developers a high "entrance fee", but also took the initiative to bear the cost of the part of the network equipment that was supposed to be borne by the developer, which brings unnecessary costs to operators. At present, Internet backbone networks have not yet achieved sufficient interconnection in China. The interests of the vast majority of Internet users can not be guaranteed. It is necessary to introduce relevant laws 
and regulations to realize the healthy development of Internet backbone networks interconnection.

\section{SUGGESTIONS FOR OPTIMIZING INTERCONNECTION BETWEEN INTERNET BACKBONE NETWORKS IN CHINA}

\section{A. Introducing transit on the premise of network security}

Since the exchange of traffic between networks is crossdomain, the dominant backbone network is vulnerable to risks when providing transit services for the vulnerable backbone network, which is why China has forbidden the interconnection mode of transit. However, with the development of the Internet and the need to enhance competitiveness, the introduction of transit can not be avoided. At present, the interconnection modes among backbone networks in China are mainly NAP and peering, and the transit is only allowed to be provided by the backbone network operators to small ISPs, is not allowed to provide for their competitors. The introduction of the transit firstly needs to solve the technical problems. It is necessary to introduce and cultivate relevant technical personnel, in order to ensure the security of network transmission in technology and form a diversified interconnection mode. According to the development experience of the backbone network in developed countries, the introduction of transit can not only save the operating costs of the vulnerable backbone networks and improve the quality of interconnection between networks, but also optimize the backbone network market structure and form a reasonable and effective competition situation from the longterm trend. This pattern, which will improve social welfare and change the current regional monopoly phenomenon between China Telecom and China Unicom, is one of the important means to solve the problems of interconnection in China.

\section{B. Reducing settlement fees and supporting the development of vulnerable backbone networks}

The special interconnection structure of Internet backbone network and development history in China, make the settlement fees between backbone networks generally higher than other countries. The settlement fees generated by the interconnection mode of NAP are regulated by the government, which strictly bases on the "Settlement Method of Internet Exchange Center". While the settlement fee for peering is decided by the backbone network itself. The settlement fee for NAP is used for reference, and the settlement fee for peering is generally not higher than NAP [8]. Currently, given the wide disparities in China's backbone network size, transit has not yet been fully introduced, and market competition is still insufficient, the government needs to increase support for vulnerable backbones, in order to solve the problem of the asymmetric market structure. Starting from the interconnection settlement of the Internet exchange center, the government should gradually reduce the settlement fee under the NAP, and develop it into a "universal service" eventually. Correspondingly, the settlement price under peering will also decrease with the settlement price of the exchange point, and the cost of small and medium-sized operators will decline, which has virtually boosted the development of the vulnerable backbone network.

\section{Establishing monitoring system of the interconnection quality}

The low interconnection quality of the Internet backbone has always been a difficult problem for the interconnection development of the backbone network. In addition to introduce the transit to increase the strength of the competition, support the vulnerable networks to narrow the scale gap, it is also a good measure to establish the interconnection quality monitoring system. In the issue of interconnection quality monitoring, Keynote Systems, a US Internet performance testing agency, has established a system to monitor the interconnection performance of the US backbone Internet, and regularly published Internet health reports online. All backbone network operators adjust themselves according to the assessment criteria, and can basically maintain the health level. In view of the actual situation in China, due to the vast territory and huge investment, it is difficult to establish monitoring bases in each province, and it is possible to concentrate funds to respectively establish monitoring stations in the eastern, central and western regions in China. The "Interim Measures for supervision and management of communication quality between Internet backbone networks" issued by the Ministry of Industry and Information Technology must be used as the monitoring basis to solve monitoring technical problems. Monitoring not only covers indicators such as packet loss rate and delay, but also analyzes various types of service packets at the application layer to monitor whether there are artificially created interconnection barriers, and guarantees communication quality from both the sending and receiving of network resources.

\section{Strengthening legislation work and adhering to government regulations}

The US backbone network is developed under the supervision of the "Anti-trust Laws" and "Competition Law", relevant laws and regulations have also continued to develop and improve. In China, in the field of Internet supervision, the government has introduced policies and regulations, such as "Measures for Administration of the Internet Information Service" and "Interim Measures for supervision and management of communication quality between Internet backbone networks". But these policies and regulations can not fully meet the needs of Internet interconnection management. Therefore, establishing and improving interconnection laws between Internet backbone networks is the focus of Internet development [9]. The legal provisions should be used to provide clear provisions for the interconnection structure, interconnected bandwidth, expansion standards, the provision of universal services and related violations, which provide the legal basis for the supervision of the government.

In terms of interconnection regulation of Internet backbone network in China, some experts and scholars have pointed out that it is necessary to deregulation. However, it is not feasible at the present stage that the development of the internet backbone network structure in China is not mature. Although the United States has applied the principle of "less intervention" for the management of the Internet, its free development atmosphere must be based on a mature development system and strict legislative foundation. The 
development of backbone network market structure, interconnection quality, interconnection mode and interconnection settlement in China also has a large gap with the United States. For the time being, it is still unable to achieve the level of deregulation. Firstly, there are only two top backbone networks in China and existing the phenomenon of "the north-south divide and conquer". The monopoly powers of them are so strong that there is no enterprise in the monopoly area that can compete with them. Secondly, the interconnection mode has not yet been completely liberalized, and the transit is not allowed, which lead to the interconnection enthusiasm of dominant backbone network is not high, and the quality of the interconnection is low. Finally, under the peering, the settlement fee is high and the settlement method is unreasonable, resulting that vulnerable networks have called for a change in settlement method. All these facts indicate that the development of backbone network in China should adhere to the government regulation. Only when the backbone market is mature and there are laws to go by, it will be possible to deregulation.

\section{REFERENCES}

[1] Ding Ke. Analysis of Internet Backbone Market Problems and Policy Suggestions in China [J]. Guangdong Communication Technology, 2007, (06):10-14.

[2] Wei Liurong. A Study on the Interconnection between Chinese Asmmetric Backbones [D]. Beijing University of Posts and Telecommunications, 2005.

[3] Yang Qingfeng. Study on Interconnection between Internet Backbone Providers [D]. Beijing University of Posts and Telecommunications, 2007.

[4] Zhang Ling. A Study on the Government Regulation of Interconnection Settlement between Internet Backbone Providers in China [D]. Jiangxi University of Finance and Economics, 2015.

[5] Sun Wei. How Do Basic Operators Improve the "Pipeline" of the Internet Backbone? -Thoughts on Interconnection of Internet Backbone Networks [J]. Telecommunications Network Technology, 2013(06):4547.

[6] Pei Wei. The Current Situation and Supervision of Internet Interconnection in China [J]. Telecommunications Network Technology, 2005(12):1-3.

[7] Zhang bo.Domestic Broadband Interoperability Problem Analysis [D].Beijing University of Posts and Telecommunications, 2013.

[8] Bai Rangrang, Wang Guangwei. Industrial Structure Change, Regulatory Inertia and Foreclosure in China Network Interconnection Market [J].China Industrial Economics, 2012(10):135-147.

[9] Zhao Liang, Yang Hua. Analysis and Suggestions on the Interconnection of Internet Backbone Network in China [J]. Communications World, 2005, (28):40. 